\title{
Incidence and size of erosions in the wrist and hand of rheumatoid patients: a quantitative microfocal radiographic study
}

\author{
J C BUCKLAND-WRIGHT AND S R WALKER
}

From the Macroradiographic Research Unit, Anatomy Department, United Medical and Dental Schools of Guy's and St Thomas's, Guy's Campus, London

SUMMARY Quantitative macroradiographic examination of a group of early to moderately advanced rheumatoid patients showed the wrist and hand to have an average of 75 (SD 26) erosions out of 142 possible sites. Joint involvement was greatest in the wrist followed by the metacarpophalangeal (MCP), proximal interphalangeal (PIP), and distal interphalangeal (DIP) joints respectively. In the wrist erosion distribution was concentrated in the radiocarpal and medial carpometacarpal complex, in the hand it tended to be located at the second and third MCP and third PIP joints. No difference was observed in erosion number and area between the right and left extremities. The distribution of the lesions is discussed in relation to the intraarticular pressures on normal hand function. The similarity of erosion development, across the joints at the different regions of the hand, suggests the presence of factors other than mechanical pressure. In general, erosions were widespread, and the largest erosions occurred in the larger bones of the wrist and hand.

The large magnification and high spatial resolution characteristic of microfocal radiography ${ }^{1}$ has led to the earlier identification of the site of erosion onset and development, together with the direct measurement of their number and size in the wrist and hand of rheumatoid patients. ${ }^{2-4}$ Our recent paper described the accuracy of measurements taken and showed that the change in size of $x$ ray features due to the disease, during the period of study, was markedly greater than that attributable to errors of measurement. ${ }^{5}$ This communication is the first of a series presenting the results of the quantitative evaluation of radiographic progression in a group of patients with early to moderately advanced rheumatoid arthritis (RA).

Based on the data collected from the first $x$ ray visit of each patient in the study, this paper describes the distribution of erosions in terms of their average incidence and area in the various regions of the wrist and hand, and gives an account of their occurrence at each of the separate bones. The extent of

Accepted for publication 28 January 1987.

Correspondence to Dr J C Buckland-Wright, Macroradiographic Research Unit, Anatomy Department, Guy's Hospital, London SE1 9RT. similarity between the right and left extremities was assessed.

\section{Patients and methods}

Forty rheumatoid patients attending the rheumatology department at this hospital were recruited for macroradiographic assessment. Patients were $x$ rayed every six months over a period of 18 months, totalling four visits. Four of these patients failed to complete the minimum requirement of three $x$ ray visits. The remaining 36 patients, of whom four were left handed, comprised 14 men and 22 women. The mean time from disease onset to the first $x$ ray was 27 months (range 3-100 months). The mean age at onset was 53 years (range 20-75 years).

The preparation of the macroradiographs, their examination and measurement have been described elsewhere. ${ }^{2-4}$ Based on the results of the macroradiographic examination of the site of onset and development of erosions, ${ }^{4}$ each bone was subdivided into sites of potential erosion development. In the wrist these sites occurred adjacent to the insertion of an interosseous ligament and at an intermediate region corresponding to that at which a subchondral erosion would develop (Fig. 1). In the 

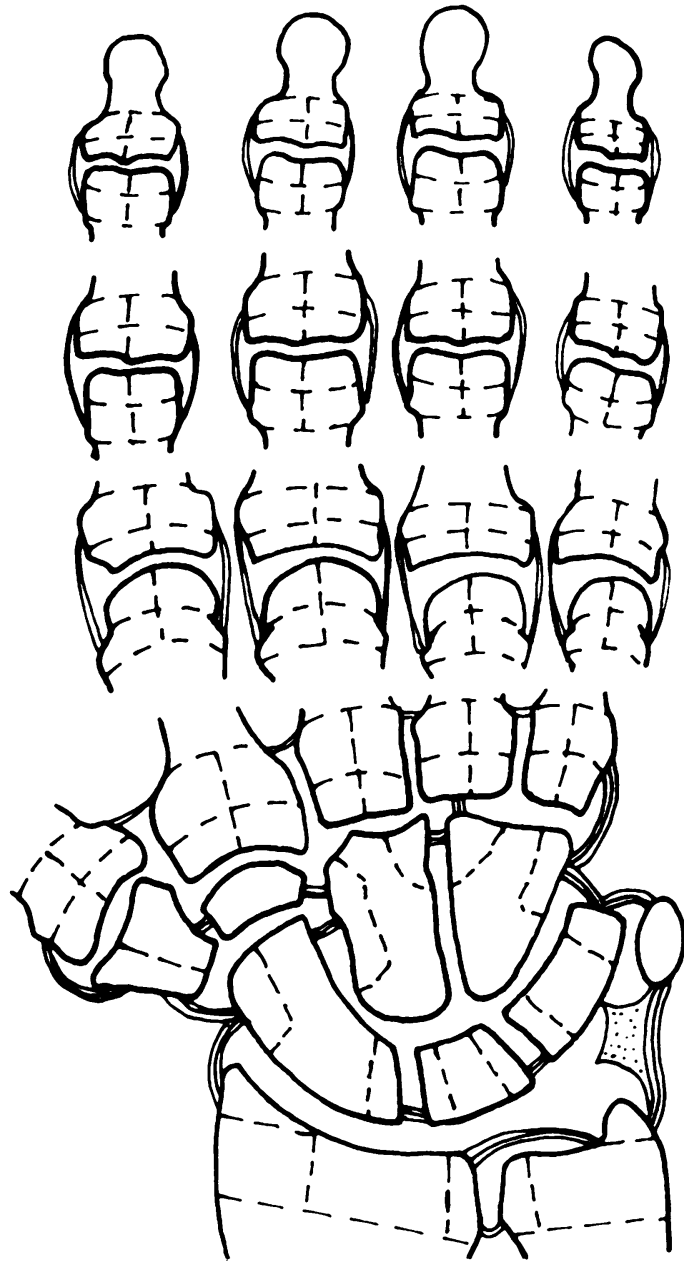

Fig. 1 Subdivisions of the bones of the wrist and hand into sites at which erosions can occur.

metacarpophalangeal (MCP), proximal interphalangeal (PIP), and distal interphalangeal (DIP) joints the bones were subdivided on the basis of erosions occurring at the juxta-articular and juxtaligamentous sites on both the lateral and medial surfaces of the bone in digits 2-5 (Fig. 1). From the radiographs of the first visit the data on the number and area of erosions at each of the 142 sites in both the right and left extremities of all patients were recorded. The data, initially recorded on microcomputer discs, were transferred to the university mainframe and concatenated into one large data file. A standard format was used in this file for each patient/hand/visit record, enabling comparison of data both within and between patients. The analysis was carried out with the SPSS-X statistics package ${ }^{6}$ and some specially written Fortran programs.
The mean number of erosions, the mean totat erosive area, and their standard deviation were calculated over the whole patient group. The joint $\$$ in the extremity were grouped into four regions: the wrist, MCP, PIP, and DIP joints. The mean number. of erosions, the mean total erosive area, and the standard deviation were calculated for each of these regions. The data were then grouped for right anf left sides and the statistics described above were repeated. The non-parametric sign test and WiE coxon signed ranks test were applied to test the hypotheses that there was no difference in the distribution of the number or area of erosion between the two extremities. To test the strength of linear association between the two hands the cor relation coefficient was calculated for total erosion number and area. The data were grouped by domia nant and non-dominant wrist and hand, and the siges test and Wilcoxon signed ranks test were used to test for any difference in the size and number of erosions between these two groups. Finally, the्d average erosion frequency and size was calculated for each of the 39 bones comprising the joints of theo wrist and hand.

\section{Results}

The mean number of erosions, the mean tota erosive area, and their standard deviations are give in Table 1 for the whole patient group, the left and right hand groups, and the separate regions of the wrist and hand. The extent of erosion development was greatest in the wrist, where erosion number contributed $40 \%$, and erosion area $56 \%$ of the totals for the whole extremity. The MCP and PIP joint had similar erosion incidence, with approximatel half of the number of erosions occurring in the wrise

Table 1 Incidence of erosions and their mean total areg in the wrist and hand, and in the separate regions

\begin{tabular}{|c|c|c|c|c|c|c|c|}
\hline \multirow{2}{*}{ Numb } & \multicolumn{2}{|c|}{ Combined } & \multicolumn{2}{|l|}{ Right } & \multicolumn{2}{|l|}{ Left } & \\
\hline & & & & & & & \\
\hline Total & $74 \cdot 5$ & $(26)^{*}$ & $73 \cdot 2$ & (26) & $75 \cdot 7$ & (26) & \\
\hline Wrist & $29 \cdot 7$ & (10) & $28 \cdot 7$ & (10) & $30 \cdot 7$ & (10) & \\
\hline MCP & $16 \cdot 6$ & (7) & $17 \cdot 2$ & (7) & 16.0 & (7) & \\
\hline PIP & $16 \cdot 1$ & (6) & $15 \cdot 3$ & (6) & $17 \cdot 1$ & (7) & \\
\hline DIP & $12 \cdot 0$ & (7) & $12 \cdot 0$ & (7) & $12 \cdot 0$ & (8) & \\
\hline \multicolumn{7}{|c|}{ Area $\left(\mathrm{mm}^{2}\right)$} & \\
\hline Total & $588 \cdot 6$ & $(435)$ & $570 \cdot 7$ & (441) & $606 \cdot 4$ & (434 & \\
\hline Wrist & $332 \cdot 6$ & (238) & $308 \cdot 1$ & (225) & 357.0 & $(251$ & \\
\hline MCP & 124.5 & (121) & 134.8 & (136) & $114 \cdot 1$ & (105 & \\
\hline PIP & $85 \cdot 4$ & (74) & $82 \cdot 3$ & (75) & 88.5 & (74 & \\
\hline DIP & $46 \cdot 2$ & (51) & $45 \cdot 6$ & (52) & $46 \cdot 8$ & (49 & \\
\hline
\end{tabular}

${ }^{*}$ Results are the mean (SD) $(n=36)$ for the right and left extremitie combined and separately. 
Radiographic study of erosions in the wrist and hand
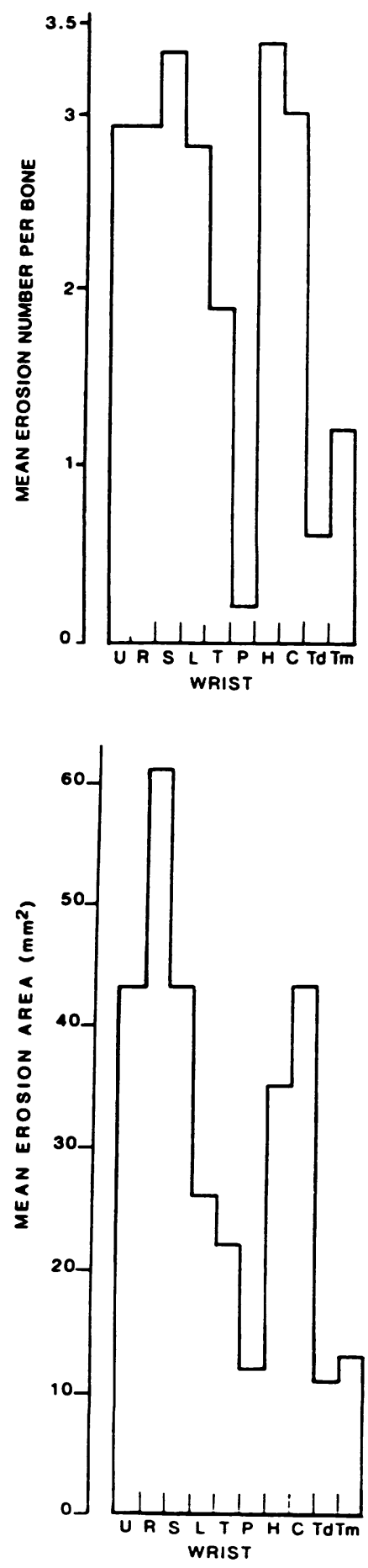

U: ULNA

R: RADIUS

S: SCAPHOID

L: LUNATE

T: TRIQUETRAL

P: PISIFORM

H: HAMATE
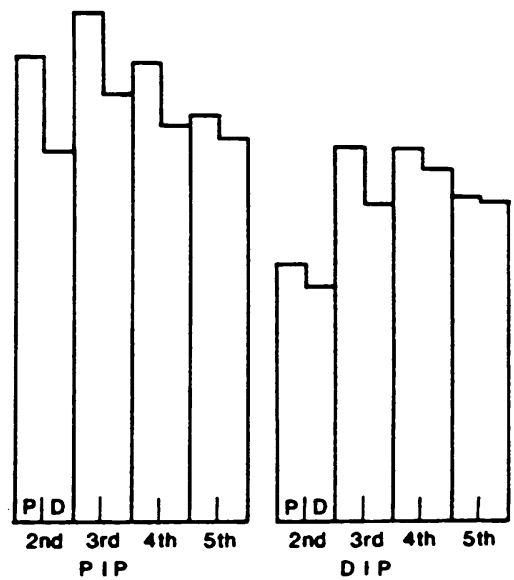

Fig. $2 \mathrm{a}$
C: CAPITATE

Td: TRAPEZOID

Tm: TRAPEZIUM

D: DISTAL

P:PROXIMAL

Fig. $2 b$

Fig. 2 Bar charts of (a) the mean number of erosions per bone and (b) the mean erosion area in the separate bones of the wrist and hand of rheumatoid patients. 
Few erosions were found in the DIP joints. The mean erosion area decreased markedly from the MCP joints ( $21 \%$ of total) to the PIP and DIP joints (14\% and $8 \%$ of the total).

No statistical difference in the mean erosion number or area could be shown between the right and left extremities. Neither the sign test nor the Wilcoxon signed ranks test rejected the hypotheses at accepted significance levels. The correlation coefficient for the total erosion number between the right and left hands was 0.911 and for the erosion area was 0.947 . The sign and Wilcoxon signed ranks tests did not reject the hypothesis of no difference in the number and size of erosions between the dominant and non-dominant hands.

The frequency of erosion formation and the mean total erosive area for each of the bones comprising the joints of the wrist and hand are presented in Fig. 2. In the wrist the largest number of erosions occurred in the scaphoid and the hamate, followed by the radius, ulna, capitate, and lunate. Significant numbers of erosions occurred also in the triquetral and the base of the fourth and fifth metacarpals. The largest size erosions were recorded in the radius, then in the ulna, scaphoid, and capitate with erosions of approximately similar dimensions, followed by those in the hamate, lunate, and triquetral. Erosion area in the remaining sites, including the base of the metacarpals, was smaller. The MCP joints showed little difference in erosion number between the joints, though the incidence in the fifth MCP joint was a little lower. The lesions were slightly larger at the distal ends of the second and third metacarpals. Erosion development in the PIP and DIP joints was similar across the joints, but a slight increase was noticeable in the third PIP joint and in the distal end of the proximal bone of most of the PIP and DIP joints.

\section{Discussion}

The macroradiographic appearance of erosions in these rheumatoid patients agrees with Resnick and Niwayama's account of lesions based on their detailed radiopathological examination of postmortem material. ${ }^{p}$ The lesions comprised small areas of radiolucency which appeared beneath the subperiosteal or subchondral bone leading to the thinning of the plate with focal discontinuity or gaps. The endosteal margin was defined by the greater radiodensity of the trabeculae bordering the lesion. The edges of an erosion thus outlined were used in the measurement of its size.

Within the limitations of standard radiographs ${ }^{3}$ Sharp et al have identified a total of 29 sites of erosion formation. ${ }^{8}$ Based on the macroradio- graphic appearance of the onset and developmen⿳亠口冋. of erosions, ${ }^{4}$ a total of 142 separate sites have bee recognised in the wrist and hand. Our presenf patient group, with early to moderately advance RA, showed an average of 75 (SD 26) erosions i욤 each wrist and hand. In addition, the results provide an assessment of the size as well as the incidence of these lesions. As seen in Table 1, the extent of erosion formation in terms of the number and area showed marked variation between patients that was in part due to differences in severity or duration of the disease, or both.

Joint involvement has been described as being greatest in the dominant side $^{9-12}$; our group of patients showed no real difference in erosion numb ber or area either between the dominant and non-dominant or between the right and left extremiw ties. The strength of linear association between the right and left hands was confirmed by the high correlation coefficient for the total area and nume ber. The differences in erosion number and are between left and right hands showed a slightly, but not statistically, increased involvement in the lepo hand in each region. These results agree with more recent observations on the absence of any significan right handed predominance of erosive changes. $\frac{1}{5}$

Joint involvement has been described as being greatest in the MCP and PIP regions, ${ }^{7}$ and othere state that the most commonly involved joints are the MCP, followed by the PIP and the wrist, with fe $\overrightarrow{\mathbb{H}}$ erosions in the DIP joints. ${ }^{910} 14$ Table 1 shows tha on average twice as many erosions were recorded i the wrist than in the MCP and PIP joints, and almosi three times as many in the wrist than in the DIE joints. The distribution of the average erosion area generally confirmed that of erosion number. The greater erosion incidence in the wrist was probab/ due to the clarity of this region in macroradios graphs compared with that recorded in standard radiographs. ${ }^{3}$ The larger number of articular suF faces, together with the larger size of the bones the wrist, could account for the average erosion are being three times greater than in the various region of the hand.

In the hand the MCP and PIP joints showe similar involvement in terms of erosion numberw though they were a much larger size in the MC $\dot{\dot{R}}$ joints. The extent of erosion incidence in the DIE joints contradicts the traditional view that they are normally spared in RA and supports the reports of their involvement. ${ }^{13} 1516$

Earlier description of the incidence of erosions in the wrist has shown that the ulna, radius, scaphoidf triquetral, and pisiform are the most severe affected and that the less common erosion sites are the lateral margins of the trapezium, capitate, and 
the base of the metacarpal bones. ${ }^{7}{ }^{17-19}$ We found erosion development in the wrist to be located in two principal regions; proximally in the ulna and radiocarpal joint and distally in the medial carpometacarpal complex. In the former the largest erosions occurred in the radius and scaphoid. Erosion frequency decreased towards the medial aspect of the wrist-namely, the scaphoid, lunate, and triquetral respectively, but in the ulna it remained similar to that of the scaphoid. In the carpometacarpal complex erosion development was greatest in the hamate and capitate, followed to a lesser extent by the base of the fifth, fourth, and third metacarpals respectively. These findings generally corroborated our previous qualitative report of erosion development, ${ }^{4}$ with the exception of the earlier underestimation of scaphoid involvement. Contrary to earlier findings ${ }^{7}$ erosion formation was observed to be markedly greater in the hamate and lower in the triquetral and pisiform. Superimposition of these last two bones in the radiographs may well account for the lower figures.

In the hand erosion development was similar across the MCP, PIP, and DIP joints. There was some agreement with previous descriptions ${ }^{9-11}$ which showed erosions concentrated in the second and third MCPs and the third PIP joints. The larger erosions observed at the distal ends of the metacarpals compared with the proximal phalanges may be attributed to the presence of a greater area of unprotected subsynovial bone adjacent to the articular cartilage. In the DIP joints marginal erosions were generally smaller and less frequent than in the PIPs, the second DIP being least affected.

Studies of hand function have shown that the greatest mechanical forces are exerted in the second and third MCP and third PIP joints, ${ }^{20}$ producing in rheumatoid patients larger erosions than at other sites. In the wrist the greatest intra-articular pressures occur during radial and ulna deviation (K Backhouse, personal communication). This would account for the predominance in our group of patients of erosions diagonally across the wrist, laterally from the radiocarpal to the medial carpometacarpal group of joints. In the hand the general uniformity of erosion development across the MCP, PIP, and DIP joints and the similarity between the dominant and non-dominant extremities indicate that mechanical factors do not produce as marked a localisation of erosion development as previously described. $^{9-1120}$ Other features could determine their extent, such as the size of the area of unprotected subsynovial bone adjacent to the articular cartilage, as seen in the different erosion area between the proximal and distal elements of the MCP joints. Additionally, there can be erosion formation adjacent to the interosseous ligaments associated with the subsynovial venous network transporting mediators from the inflamed synovium. ${ }^{4}$ In conclusion, erosions were found to be widespread, supporting recent observations, ${ }^{16}$ and in patients with early to moderately advanced disease the largest erosions occurred in the larger bones of the wrist and hand.

We wish to thank Dr R Grahame, Professor G Panayi, and Dr T Gibson for their cooperation in providing the patients for $x$ ray examination. This work is being generously supported by the Arthritis and Rheumatism Council.

\section{References}

1 Buckland-Wright J C. Qualitative and quantitative assessment of tissue organisation in normal and diseased organs. In: Ely R V. ed. Microfocal radiography. London, New York: Academic Press, 1980: 147-95.

2 Buckland-Wright $\mathrm{J} \mathrm{C}$. X-ray assessment of activity in rheumatoid disease. $\mathrm{Br} J$ Rheumatol 1983; 22: 3-10.

3 Buckland-Wright $\mathrm{J} \mathrm{C}$. Advances in the radiological assessment of rheumatoid arthritis. Br J Rheumatol 1983; 22 (suppl): $34-43$.

4 Buckland-Wright J C. Microfocal radiographic examination of erosions in the wrist and hands of patients with rheumatoid arthritis. Ann Rheum Dis 1984; 43: 160-71.

5 Buckland-Wright J C, Carmichael I, Walker S R. Quantitative microfocal radiography accurately detects joint changes in rheumatoid arthritis. Ann Rheum Dis 1986; 45: 379-83.

6 SPSS-X Inc. SPSS- $X$ user's guide. New York: McGraw-Hill, 1983.

7 Resnick D, Niwayama G. Diagnosis of bone and joint disorders. Vol II. Philadelphia: Saunders, 1981: 906-1007.

8 Sharp J J. Lidsk M D, Collins L C. Moreland J. Methods of scoring the progression of radiological changes in rheumatoid arthritis. Arthritis Rheum 1971; 14: 706-20.

9 Fleming A. Crown J M, Corbett M. Early rheumatoid disease. Patterns of joint involvement. Ann Rheum Dis 1976; 35: 361-4.

10 Fleming $\mathrm{A}$, Crown $\mathrm{J} \mathrm{M}$, Corbett $\mathrm{M}$. Incidence of joint involvement in early rheumatoid arthritis. Rheumatol Rehabil 1976; 15: 92-6.

11 Mattingly P C, Matheson J A, Dickson R A. The distribution of radiological joint damage in the rheumatoid hand. Rheumatol Rehabil 1979; 18: 142-7.

12 Owsianik W D J, Kundi A, Whitehead N, Kraag G R, Goldsmith C. Radiological articular involvement in the dominant hand in rheumatoid arthritis. Ann Rheum Dis 1980; 39: 508-10.

13 Halla J T, Fallahi S, Hardin J G. Small joint involvement: a systematic roentgenographic study in rheumatoid arthritis. $A n n$ Rheum Dis 1986: 45: 327-30.

14 Brook A. Corbett M. Radiographic changes in early rheumatoid disease. Ann Rheum Dis 1977; 36: 71-3.

15 Campion G, Dieppe P. Watt I. Heberden's nodes in osteoarthritis and rheumatoid arthritis. Br Med $J$ 1983; 287: 1512.

16 Scott D L, Coulton B L. Popert A J. Long term progression of joint damage in rheumatoid arthritis. Ann Rheum Dis 1986; 45: 373-8.

17 Martel W. The pattern of rheumatoid arthritis in the hand and wrist. Radiol Clin North Am 1964; 2: 221-34.

18 Martel W, Nays J T, Duff I F. The pattern of bone erosion in the hand and wrist in rheumatoid arthritis. Radiology 1965; 84: 204-14.

19 Resnick D. Rheumatoid arthritis of the wrist: the compartmental approach. Med Radiogr Photogr 1976; 52: 50-88.

20 Glick E N. Influence of mechanical factors on the rheumatoid wrist. Proc $R$ Soc Med 1966; 59: 555-8. 